\title{
Review of: "Compound jetting from bubble bursting at an air-oil-water interface"
}

Jianlin Liu

Potential competing interests: The author(s) declared that no potential competing interests exist.

The authors have reported an intesesting phenomenon. They found that bubble bursting can disperse highly viscous surface organic matter as compound jet droplets into air. Then they unravelled the physical mechanism related to the jetting dynamics at an oil-covered aqueous surface. They performed powerful experiments, and the elegant scaling laws were made. The analysis is very detailed. These findings may advance the insights into the interfacial dynamics of complex fluids, as well as the size evaluation for oily aerosol generated by bursting bubbles regarding marine environment and public health. 